\title{
Comparison of T-Cell Interferon- $\gamma$ Release Assays for Mycobacterium tuberculosis-Specific Antigens in Patients with Active and Latent Tuberculosis
}

\author{
Yoshihiro Kobashi $\cdot$ Hiroki Shimizu $\cdot$ Yoshihiro Ohue $\cdot$ \\ Keiji Mouri · Yasushi Obase $\cdot$ Naoyuki Miyashita • \\ Mikio Oka
}

Received: 15 July 2009/Accepted: 18 March 2010/Published online: 27 April 2010

(c) The Author(s) 2010. This article is published with open access at Springerlink.com

\begin{abstract}
Through the use of QuantiFERON-TB Gold, a commercial IFN- $\gamma$ assay, we compared differences in quantitative T-cell responses to Mycobacterium tuberculosis (MTB)-specific antigens [QuantiFERON TB-2G (QFT-2G)] between patients with active tuberculosis (TB) disease and those with latent TB infection (LTBI). The patient group consisted of 180 patients with active TB disease (culture-positive for MTB) and 50 screening contacts with LTBI-positive response to the QFT-2G test. We prospectively performed a tuberculin skin test (TST) and a QFT-2G test for all subjects. The median IFN- $\gamma$ levels upon the application of both antigens, ESAT-6 and CFP-10, were significantly higher in patients with active TB disease than in those with LTBI. A combined positive response to both antigens occurred at a higher rate in patients with active TB disease than in those with LTBI. There were no significant relationships between the quantitative responses of IFN- $\gamma$ to both antigens and the maximum induration on TST in both patient groups. We demonstrated significant differences in the quantitative responses of IFN- $\gamma$ to MTB between patients with active TB disease and those with LTBI in this study. However, there was an overlap in the IFN- $\gamma$ levels between active TB disease and LTBI groups. Therefore, it would be difficult to use the QFT-2G test to completely discriminate active TB disease from LTBI.
\end{abstract}

Y. Kobashi $(\bowtie) \cdot$ H. Shimizu · Y. Ohue · K. Mouri •

Y. Obase - N. Miyashita - M. Oka

Division of Respiratory Diseases, Department of Medicine, Kawasaki Medical School, 577 Matsushima, Kurashiki, Japan e-mail: yoshihiro@med.kawasaki-m.ac.jp
Keywords Active tuberculosis (TB) disease . Latent tuberculosis infection (LTBI) - Tuberculin skin test (TST) · QuantiFERON TB-2G (QFT-2G)

\section{Introduction}

The development of T-cell-based interferon-gamma (IFN- $\gamma$ ) assays (IGRAs) for Mycobacterium tuberculosis (MTB)specific antigens represents a significant advance that is expected to replace the tuberculin skin test (TST) in screening for active TB disease or latent tuberculosis infection (LTBI) [1-3]. These IGRAs have demonstrated excellent specificity and shown a closer correlation with the exposure gradient than the TST [4-6]. However, it is generally assumed that these tests, like TST, cannot discriminate between active TB disease and LTBI. There are some limitations to the use of IGRAs in serial testing. The utility of these assays for monitoring disease activity and treatment response, or as predictors of the progression of LTBI to active TB disease, is currently the subject of ongoing research.

Two commercial IGRAs have recently become available, QuantiFERON-TB Gold In-Tube(Cellestis Ltd., Victoria, Australia) with ELISA used to measure IFN- $\gamma$ concentrations in supernatants, and the T-SPOT.TB test (Oxford Immunotec, Oxford, UK) with an enzyme-linked immunospot (ELISPOT) assay used to detect individual $\mathrm{T}$ cells producing IFN- $\gamma[1,7]$. QuantiFERON TB-2G (QFT$2 \mathrm{G})$ is commonly used for the diagnosis of active TB disease or LTBI, but T-SPOT.TB or QFT Gold In-Tube has not yet been approved in Japan. We therefore compared quantitative T-cell responses to MTB-specific antigens between cohorts of contacts with LTBI and patients with active TB disease using the commercial QFT-2G test. 


\section{Materials and Methods}

Study Population

One hundred eighty patients confirmed to be positive for MTB on culture of sputum, bronchoalveolar lavage fluid, peritoneal fluid, or lymph node tissue samples and a cohort of 50 screening contacts with LTBI (positive QFT-2G response with or without a positive TST result) drawn from the community were prospectively enrolled starting in January 2005. Screening contacts with LTBI all had exposure histories to their index case 8-12 weeks earlier and all index cases were smear- and culture-positive for MTB using sputum specimens. However, none of these subjects with LTBI showed any clinical symptoms (fever, cough, or sputum), abnormal inflammatory responses, or abnormal radiological findings suggesting pulmonary TB. This study was approved by the institutional ethical committee at each hospital. Patients were diagnosed at Kawasaki Medical School Hospital and four affiliated hospitals. All patients with active TB disease showed negative findings on enzyme-linked immunosorbent assay (ELISA) testing for HIV. Regarding subjects screened for contact with the index case, HIV testing was not performed, but subjects with a history of HIV infection were excluded from data analyses. Demographic data from patients with active TB disease and screening contacts with LTBI were collected, including any history of previous TB or antituberculous treatment, other underlying diseases, and administration of immunosuppressive drugs within 3 months before enrolment in this study.

\section{Sample Collection and TST}

A heparinized blood sample was collected by venipuncture from each patient for whole-blood IFN- $\gamma$ assay. Blood samples were collected before administration of the Mantoux TST. For the TST, $0.1 \mathrm{ml}$ of tuberculin PPD [Nippon $\mathrm{BCG}$, equivalent to three tuberculin units (TU) of purified protein derivative solution (PPD-S)] was injected intradermally into the volar aspect of the forearm, and the transverse induration diameter was measured $48 \mathrm{~h}$ later. The results of the test were interpreted by hospital staff based on the patient's degree of risk, according to current guidelines [8]. A lower cutoff of $5 \mathrm{~mm}$ for a positive test was used for individuals.

\section{QFT-2G Test}

The QFT-2G test was performed according to the manufacturer's recommendations, and the test result was judged according to CDC guidelines [1]. The test result was considered positive if the IFN- $\gamma$ level in the sample well after stimulation with ESAT-6 and/or CFP-10 was $\geq 0.35 \mathrm{IU} / \mathrm{ml}$, irrespective of the result for the positive control well. The test was considered negative if the IFN- $\gamma$ level was $<0.35 \mathrm{IU} / \mathrm{ml}$ and the IFN- $\gamma$ level of the positive control well was $\geq 0.35 \mathrm{IU} / \mathrm{ml}$. The test result was considered indeterminate if the IFN- $\gamma$ level was $<0.35 \mathrm{IU} / \mathrm{ml}$ in both antigen wells and $<0.5 \mathrm{IU} / \mathrm{ml}$ in the positive control well. The QFT-2G test was performed for all patients before initiating the administration of antituberculous drugs.

\section{Statistical Analysis}

Data were input into Excel 2000 (Microsoft Corp., Redmond, WA) and then transferred to Santa software, version 7.0 (Santa, Vienna, Austria), for statistical analysis. Pearson's $\chi^{2}$ test was used to compare data from the two groups and McNemar's test was used to compare paired data. The Mann-Whitney $U$ test was used to compare unpaired data. The level of significance was $P<0.05$.

\section{Results}

One hundred thirty subjects screened after contact with index cases were included in this study protocol. They consisted of 70 males and 60 females, and the mean age was $41.8 \pm 9.8$ years (range $=18-76$ ). The previous history of TST was unreliable in most subjects. Ninety subjects $(69 \%)$ had a history of BCG vaccination. The comparison of TST and QFT-2G test results for all contacts screened is shown in Table 1. The TST induration diameter was larger than $5 \mathrm{~mm}$ in $78(60 \%)$ subjects, and $50(38 \%)$ showed positive QFT-2G test results. While both TST and QFT-2G tests were positive in 34 screening contacts (26\%), only the QFT-2G test was positive in $16(12 \%)$ and only the TST was positive in $44(34 \%)$. Of all screening contacts, 50 subjects $(38 \%)$ fulfilled the inclusion criteria for LTBI in this study.

A comparison of TST and QFT-2G test results for patients with active TB disease is shown in Table 2. The positive response rate on TST did not significantly differ between all subjects screened after contact (60\%) and those

Table 1 Comparison of TST and QFT-2G test results for all contacts screened (130 cases)

\begin{tabular}{llcc}
\hline QFT-2G test & \multicolumn{3}{l}{ TST } \\
\cline { 2 - 4 } & Positive & Negative & Total \\
\hline Positive & $34(26 \%)$ & $16(12 \%)$ & $50(38 \%)$ \\
Negative & $44(34 \%)$ & $31(24 \%)$ & $75(58 \%)$ \\
Indeterminate & 0 & $5(4 \%)$ & $5(4 \%)$ \\
Total & $78(60 \%)$ & $52(40 \%)$ & $130(100 \%)$ \\
\hline
\end{tabular}

TST tuberculin skin test, $Q F T-2 G$ QuantiFERON TB-2G 
Table 2 Comparison of TST and QFT-2G test results for patients with active TB disease (180 cases)

\begin{tabular}{llll}
\hline QFT-2G test & \multicolumn{2}{l}{ TST } & \\
\cline { 2 - 4 } & Positive & Negative & Total \\
\hline Positive & $114(63 \%)$ & $36(20 \%)$ & \\
Negative & 0 & $11(6 \%)$ & \\
Indeterminate & 0 & $19(11 \%)$ & \\
Total & $114(63 \%)$ & $66(37 \%)$ & \\
\hline
\end{tabular}

TST tuberculin skin test, $Q F T-2 G$ QuantiFERON TB-2G

with active TB disease (63\%). The positive response rate to the QFT-2G test was significantly higher in patients with active TB disease $(83 \%)$ than in all screening contacts $(38 \%)$. The positive response rate to the QFT-2G test $(83 \%)$ was significantly higher than to the TST $(63 \%)$ in patients with active TB disease. The positive response rate to the QFT-2G test (38\%) was significantly lower than to the TST $(60 \%)$ in all screening contacts.

In 180 patients with active TB disease, acid-fast smear results of samples were positive in 93 patients (52\%) and negative in the remaining 87 patients.

The clinical characteristics of the 50 screening contacts with LTBI and the 180 patients with active TB disease are shown in Table 3. There were no significant differences in the clinical characteristics between LTBI and active TB disease patients except for age, sex, and underlying disease including respiratory disease.

Concerning the comparison of QFT-2G test results related to combined and separate responses to ESAT- 6 and CFP-10 antigens for LTBI and active TB disease, the positive response rate for both ESAT-6 and CFP-10 antigens was significantly lower in community contacts with LTBI than that in patients with active TB disease. The positive response rates for ESAT- 6 only and for CFP-10 only were lower in patients with active TB disease than in screening contacts with LTBI (Table 4).

The IFN- $\gamma$ level in the response to ESAT-6 and CFP-10 antigens for screening contacts with LTBI and patients with active TB disease is shown in Table 5. The IFN- $\gamma$ levels for ESAT-6 and CFP-10 antigens were significantly higher in patients with active TB disease (IFN- $\gamma$ level for ESAT-6: $1.24 \pm 0.30 \mathrm{IU} / \mathrm{ml}$, IFN- $\gamma$ level for CFP-10: $1.58 \mathrm{IU} / \mathrm{ml}$ ) than in screening contacts with LTBI (IFN- $\gamma$ level for ESAT-6: $0.61 \pm 0.20 \mathrm{IU} / \mathrm{ml}$, IFN- $\gamma$ level for CFP-10: $0.72 \pm 0.21 \mathrm{IU} / \mathrm{ml})$.

\section{Discussion}

We noted a significant reduction in the positive response rate for both ESAT-6 and CFP-10 antigens in screening contacts with LTBI than in patients with active TB disease, and the quantitative T-cell responses to ESAT- 6 and CFP10 antigens were significantly higher in patients with active TB disease than in those with LTBI using the QFT-2G assay.

In previous reports, Janssens et al. [9] and Chee et al. [10] reported a significant difference in the quantitative values of T-SPOT.TB responses between active TB cases and contacts with positive T-SPOT.TB responses, with a large overlap in the range of values. Although Chee et al. [10] did not identify a significant difference in the quantitative values using the QFT Gold-In-Tube assay in previous reports, we found a significant difference in quantitative QFT-2G responses between the two groups in this study, consistent with the findings in a previous report by Higuchi et al. [11]. Higuchi et al. [11] also indicated that the geometric mean of the IFN- $\gamma$ production level in the active TB disease group was significantly larger than that in the LTBI group, and the combined parameters of ESAT6 and CFP-10 significantly contribute to the active TB disease risk for infected subjects using the QFT-2G test. Differences in the sensitivity of T-SPOT.TB and QFT-2G assays in immunocompromised patients have been well reported [4]. Although both assays measure T-cell IFN- $\gamma$ responses to the Mycobacterium tuberculosis (MTB)-specific antigens ESAT-6 and CFP-10 after overnight stimulation, the measurement of IFN- $\gamma$ levels in whole blood in the QFT-2G assay [as opposed to measuring the number of IFN- $\gamma$-producing $\mathrm{T}$ cells in a standardized number of peripheral blood mononuclear cells (PBMCs) using T-SPOT.TB] may render this assay less sensitive than T-SPOT.TB. Regardless of this hypothesis, there was a significant difference in quantitative QFT-2G responses between active TB cases and screening contacts with LTBI in this study.

While the median IFN- $\gamma$ level in response to ESAT-6 and CFP-10 antigens in patients with active TB disease was more than two times higher than that of screening contacts with LTBI, the large overlap in the range of values between these two groups suggests that isolated QFT-2G results would not be useful as a diagnostic method to discriminate between active TB disease and LTBI.

There are several limitations to this study. First, because there is no gold standard for the diagnosis of LTBI, cases with active TB disease may have been missed among screening contacts with presumed LTBI. However, this is highly unlikely since all of the subjects demonstrated normal clinical and radiological findings. Indeed, there were no missed cases of active TB disease detected during the subsequent 3 years of follow-up.

Second, because there were significant differences in the frequency of patients with underlying disease and mean age of both groups (Table 3), these factors have influenced 
Table 3 Clinical

characteristics of 50 screening contacts with latent tuberculosis infection (LTBI) and 180 patients with active tuberculosis (TB) disease
${ }^{a}$ With hemodialysis

b All patients received corticosteroid therapy

\begin{tabular}{llll}
\hline Clinical characteristics & LTBI $(n=50)$ & $\begin{array}{l}\text { Active TB disease } \\
(n=180)\end{array}$ & $P$ value \\
\hline Age (years) (mean $\pm \mathrm{SD})$ & $41.6 \pm 9.5$ & $63.8 \pm 10.6$ & 0.010 \\
Sex (Male:Female) & $24: 26$ & $140: 40$ & 0.022 \\
Smoking history & $30(60 \%)$ & $120(67 \%)$ & 0.768 \\
Alcohol abuse history & $6(12 \%)$ & $32(18 \%)$ & 0.356 \\
Underlying disease & $18(36 \%)$ & $133(74 \%)$ & 0.035 \\
Respiratory disease & $5(12 \%)$ & $61(34 \%)$ & 0.038 \\
Healed pulmonary TB & $0(0 \%)$ & $20(11 \%)$ & 0.008 \\
COPD & $2(4 \%)$ & $13(7 \%)$ & 0.320 \\
Others & $4(8 \%)$ & $28(16 \%)$ & 0.101 \\
Nonrespiratory disease & $13(26 \%)$ & $72(40 \%)$ & 0.070 \\
Gastrointestinal disease & $4(8 \%)$ & $18(10 \%)$ & 0.755 \\
Diabetes mellitus & $3(6 \%)$ & $15(8 \%)$ & 0.842 \\
Cancer & $1(2 \%)$ & $14(8 \%)$ & 0.084 \\
Cardiovascular disease & $3(6 \%)$ & $13(7 \%)$ & 0.893 \\
Collagen vascular disease & $1(2 \%)$ & $7(4 \%)$ & 0.396 \\
Chronic renal failure & $0(0 \%)$ & $5(3 \%)$ & 0.108 \\
Immunosuppressive treatment ${ }^{\mathrm{b}}$ & $1(2 \%)$ & $13(10 \%)$ & 0.061 \\
Received BCG vaccination & $34(63 \%)$ & $116(64 \%)$ & 0.867 \\
Received TB treatment & $0(0 \%)$ & $29(16 \%)$ & 0.008 \\
Laboratory data & $4620 \pm 543$ & $4058 \pm 518$ & 0.350 \\
WBC count (cells/ $\mu \mathrm{l})$ & $1036 \pm 92$ & $6.4 \pm 0.8$ & 0.402 \\
Lymphocyte count $($ cells/ $\mu \mathrm{l})$ & $6.9 \pm 1.0$ & $3.3 \pm 0.6$ & 0.346 \\
Total protein $(\mathrm{g} / \mathrm{dl})$ & $3.7 \pm 0.8$ & & 0.367 \\
Albumin $(\mathrm{g} / \mathrm{dl})$ & & &
\end{tabular}

Table 4 Comparison of the QFT-2G test results related to the combined and separate responses to ESAT-6 and CFP-10 antigens for patients with LTBI and active TB disease

\begin{tabular}{lccr}
\hline QFT-2G test & LTBI $(n=50)$ & $\begin{array}{l}\text { Active TB disease } \\
(n=180)\end{array}$ & $P$ value \\
\hline ESAT-6 only positive & $22(44 \%)$ & $49(27 \%)$ & 0.077 \\
CFP-10 only positive & $24(48 \%)$ & $51(28 \%)$ & 0.048 \\
ESAT-6 and CFP-10 positive & $4(8 \%)$ & $50(28 \%)$ & 0.026 \\
Total & $50(100 \%)$ & $150(83 \%)$ & 0.564 \\
\hline
\end{tabular}

QFT-2G QuantiFERON TB-2G

Table 5 IFN- $\gamma$ level (U/ml) in response to ESAT-6 and CFP-10 antigens for patients with LTBI and active TB disease

\begin{tabular}{llll}
\hline QFT-2G test & LTBI $(n=50)$ & Active TB disease $(n=180)$ & $P$ value \\
\hline ESAT-6 & $0.61 \pm 0.20(0.35-4.25)$ & $1.24 \pm 0.30(0.20-10.62)$ & 0.010 \\
CFP-10 & $0.72 \pm 0.21(0.35-4.60)$ & $1.58 \pm 0.36(0.24-11.90)$ & 0.008 \\
\hline
\end{tabular}

QFT-2G QuantiFERON TB-2G

the quantitative response of IFN- $\gamma$ for both MTB-specific antigens in this study.

Third, the present definition of LTBI is arbitrary. Indeed, there is no gold standard for defining LTBI. IGRA results correlate better with exposure than TST results; the lifetime risk of active TB disease based on IGRA results only currently remains unknown [12]. The clinical situation resulting from the present $\mathrm{CDC}$ recommendations was investigated [1]; subjects considered as having probable LTBI were detected by a positive QFT-2G test result 
despite negative TST results. Although the T-SPOT.TB test has been used as an IGRA, Janssens et al. [9] indicated that studying only T-SPOT.TB-positive subjects or T-SPOT.TBand TST-positive subjects did not significantly alter the results according to prevailing guidelines.

Fourth, we used the QFT-2G test as an IGRA in this study because the QFT-2G assay is currently the only IGRA commercially available in Japan. Newer commercial assays such as T-SPOT.TB (Oxford-Immunotec) and QuantiFERON-TB Gold In-Tube (Cellestis) have many differences and may provide higher levels of sensitivity [13]. Indeed, Harada et al. [14] reported that the sensitivity of the QFT Gold In-Tube test $(93 \%)$ was significantly higher than that of the QFT-2G test $(81 \%)$, although the specificities of both tests were the same (99\%). We think that the QFT Gold In-Tube or T-SPOT.TB should be approved and made commercially available for the supportive diagnosis of active TB disease and LTBI.

In conclusion, we demonstrated significant differences in the quantitative responses of IFN- $\gamma$ to MTB between patients with active TB disease and those with LTBI in this study. However, there is an overlap in the IFN- $\gamma$ levels between active TB disease and LTBI groups. Therefore, it would be difficult to use the QFT-2G test to completely discriminate active TB disease from LTBI. A large-scale longitudinal study that investigates this important potential use of IGRAs test is awaited.

Open Access This article is distributed under the terms of the Creative Commons Attribution Noncommercial License which permits any noncommercial use, distribution, and reproduction in any medium, provided the original author(s) and source are credited.

\section{References}

1. Mazurek GH, Jereb J, Lobue P, Iademarco MF, Metchoch B, Vernon A, Division of Tuberculosis Elimination, National Center for HIV, STD, and TB Prevention, Centers for Disease Control and Prevention (CDC) (2005) Guidelines for using the QuantiFERON-TB gold test for detecting mycobacterium tuberculosis infection, United States. MMWR Recomm Rep 54(RR-15):49-56
2. Pai M, Kalantri S, Dheda K (2006) New tools and emerging technologies for the diagnosis of tuberculosis. Latent tuberculosis. Expert Rev Mol Diagn 6:413-422

3. Lalvani A (2007) Diagnosing tuberculosis infection in the $21 \mathrm{st}$ century. New tools to tackle an old enemy. Chest 131:1898-1906

4. Menzies D, Pai M, Comstock G (2007) Meta-analysis: new tests for the diagnosis of latent tuberculosis infection: areas of uncertainty and recommendations for research. Ann Intern Med $146: 340-354$

5. Ewer K, Deeks J, Alvarez L, Waller S, Andersen P, Monk P, Lalvani A (2003) Comparison of T-cell-based assay with tuberculin skin test for diagnosis of Mycobacterium tuberculosis outbreak. Lancet 361:1168-1173

6. Arend SM, Thijsen SF, Leyten EM, Bouwman JJ, Franken WP, Koster BF, Cobelens FG, van Houte AJ, Bossink AW (2007) Comparison of two interferon-gamma assays and the tuberculin skin test for tracing tuberculosis contacts. Am J Respir Crit Care Med 175:618-627

7. Rothel JS, Andersen P (2005) Diagnosis of latent Mycobacterium tuberculosis infection: is the demise of the Mantoux test imminent? Expert Rev Anti Infect Ther 3:981-993

8. American Thoracic Society (2000) Targeted tuberculin testing and treatment of latent tuberculosis infection. MMWR Recomm Rep 49(6): 1-51

9. Janssen JP, Roux-Lombard P, Perneger T, Metzger M, Vivien R, Rochat $\mathrm{T}$ (2007) Quantitative scoring of an interferon- $\gamma$ assay for differentiating active from latent tuberculosis. Eur Respir $\mathbf{J}$ 30:722-727

10. Chee CB, Barkham TM, KhinMar KW, Gan SH, Wang YT (2009) Quantitative T-cell interferon-gamma responses to Mycobacterium tuberculosis-specific antigens in active and latent tuberculosis. Eur J Clin Microbiol Infect Dis 28(6):667-670

11. Higuchi K, Harada N, Fukazawa K, Mori T (2008) Relationship between whole-blood interferon-gamma responses and the risk of active tuberculosis. Tuberculosis 88:244-248

12. Shams H, Weis SE, Klucar P, Lalvani A, Moonan PK, Pogoda JM, Ewer K, Barnes PF (2005) Enzyme-linked immunospot and tuberculin skin testing to detect latent tuberculosis infection. Am J Respir Crit Care Med 172:1161-1168

13. Richeldi L (2006) An update on the diagnosis of tuberculosis infection. Am J Respir Crit Care Med 174:736-742

14. Harada N, Higuchi K, Yoshiyama T, Kawabe Y, Fujita A, Sasaki Y, Horiba M, Mitarai S, Yonemaru M, Ogata H, Ariga H, Kurashima A, Wada A, Takamori M, Yamagishi F, Suzuki K, Mori T, Ishikawa N (2008) Comparison of the sensitivity and specificity of two whole blood interferon-gamma assays for M. tuberculosis infection. J Infect 56:348-353 